\title{
Multi-Camera-based High Precision Measurement Approach for Surface Acquisition
}

\section{Takeshi Hashimoto, Takayuki Suzuki}

Shizuoka University, Dept. of Electrical \& Electronics Engineering, 5-1, 3-chome Johoku, Naka-ku, Hamamatsu, 432-8561, Japan

tethash@ipc.shizuoka.ac.jp, f0230108@ipc.shizuoka.ac.jp

\section{Hidemichi Aoshima}

SUZUKI Motor Corporation, 300 Takatsuka-cho, Minami-ku, Hamamatsu, 4328611, Japan

\section{András Rövid}

Óbuda University, John von Neumann Faculty of Informatics, Bécsi út 96/B, 1034 Budapest, Hungary, rovid.andras@ @ik.uni-obuda.hu

\footnotetext{
Abstract: In this paper, a unique 3D shape measurement method for measurement of nearly plain car body parts is proposed. The main advantage of the system is the high accuracy (about $0.1 \mathrm{~mm}$ ), the high flexibility and the real-time processing. The primary application of the method is the 100\% inspection of press formed car parts in the factory since in these cases the high accuracy and the real-time feature are indispensability. In order to achieve suitable measurement accuracy the camera specifications were carefully inspected. As result, a new type of gray-level marker and a new technique was proposed by the authors for compensating the time variability of marker central points in the camera images. In order to achieve real-time processing, the correspondence matching was devised. Furthermore, since the proposed method is based on processing camera images, i.e. the detection of feature-points on the target (such as screw holes) is also supported. The measured feature-points can easily be compared to CAE data.
}

Keywords: 3D shape measurement; real-time; high-precision; car parts 


\section{Introduction}

High precision 3D measurement stands for a key issue in many applications. Although there are various kind of 3D measurement devices on the market, their improvement and adaptation to the requirements of specific applications are highly welcome as for instance for vehicle frame and vehicle body inspection, turbine blade inspection [12], for various kind of medical applications [11], etc.

As few examples of most widely used 3D measurement approaches utilizing image data, the structured light based ones as well as methods based on laser strip projection and fringe analysis [1] [2] [4] can be mentioned. Furthermore, there are also various hardware based solutions, e.g. Time of Flight (TOF) cameras [3] [10], as well as approaches based on stereo imaging without the use of pattern projection. The main bottleneck of the latter approach is the accuracy of the correspondence point-matching. The utilization areas of such systems are especially those ones which do not require high accuracy and robustness. Since all the existing realizations differ in several features, their utilization depends on the application requirements. Beside the above mentioned techniques one can find in the literature other interesting approaches, as well [9].

The primary aims related to the development of 3D measurement techniques are to achieve high accuracy as well as to ensure quick and easy measurement. To achieve high accuracy many factors have to be taken into account during the development, e.g. CCD (CMOS) image sensor noise, lens distortion, working distance, image resolution, quantization error of image sensors, etc. The latter one was considered as an unavoidable error in 3D measurement and has been studied and modeled by the authors. Although the error caused by the quantization is not reasonable in general, by high precision measurement its impact may be significant, therefore its reduction plays crucial role. The influence of the other above mentioned error sources may be reduced and compensated by various known techniques [6-8].

This paper describes a unique 3D shape measurement method. In the framework of the research, the main aim was to achieve an accuracy of few dozen microns and ensure the real-time measurement based on pattern projection combined with a multi-camera system. Furthermore, an additional objective was to ensure the acquisition of large objects - targets having the size of a car door or even larger at once by maintaining the accuracy and measurement speed at similar level (the minimum of the achieved measurement error was $0.05 \mathrm{~mm}$ ). The impact of the quantization error has been taken into account which has been efficiently reduced by a new technique described later in the upcoming sections. The primary application of the proposed approach is the $100 \%$ inspection of the press formed car parts in the factories. Furthermore, the method can advantageously be applied to observe variations on the cliff during the motion of the vehicle. Trough the analyses of the obtained time series, the short-term failure of the cliff may be predicted. In these cases, the high accuracy and the real-time measurement are indispensable. 
Conventional 3D shape measurement systems produce dense point clouds. By the proposed method, the feature-points of the target - such as screw holes - may also be detected. This feature of the system makes it possible to compare the results of the measurement to CAE (Computer Aided Engineering) data.

During the development of the system - in order to achieve the required measurement accuracy - , the following issues have been studied:

Since the proposed system is based on projecting markers onto the targeted surface acquired by a multi-camera system (see later in the upcoming sections), the characteristics of the markers were crucial. Numerous analysis have been performed to find the most suitable (most robust to noise) marker. An important consideration during the analysis was the careful inspection of the camera specifications. Based on the performed investigation a new type of gray level marker was proposed together with a new compensation technique aimed for compensating the time variability (due to different type of noise sources) of marker locations in the camera images. Furthermore, to speed up the processing the searching method for corresponding points was devised.

The paper is organized as follows: In Section 2 the architecture and working principle of the proposed 3D measurement system is described; Section 3 shows the achieved results and their evaluation. In Section 4 conclusions and future works are reported.

\section{The Proposed System}

\subsection{Illustration of the Proposed System}

Fig. 1 illustrates the architecture of the proposed 3D measurement system. Its main components are four high-resolution cameras and a projector to project a pattern composed of gray level markers onto the surface of the target. The primary application of the system is strongly related to the inspection of vehicle body parts during the various stages of their design and production. The main requirement was the real-time, high accuracy 3D measurement of car body parts such as car door or engine hood. In order to achieve high precision and real-time processing, the following issues have been considered:

-Improvement of the applied markers to reduce the error caused by the image sensor noise

-Compensation of errors caused by camera distortions

-Fast corresponding point search to support the real-time measurement

In the upcoming sections, the corresponding techniques are described in detail. 


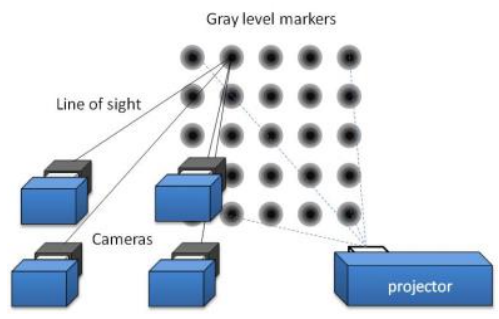

Figure 1

Components of the proposed system and the projected grid of gray level markers

\subsection{Improvement of the "G-Marker"}

In order to ensure precise detection of marker central points, many factors have to be taken into account, e.g. CCD (CMOS) image sensor noise, resolution, lens distortion, etc. When capturing the projected markers by camera, due to the mentioned factors the real properties of the markers may be corrupted - their shape properties for instance - and therefore it becomes difficult to accurately determine their exact central point. Usually the markers can easily be extracted by thresholding, however in case of a simple circular pattern - due to the above factors - the estimated center of gravity will not correspond to the exact central point of the marker. Although the related error is not reasonable, it is still important to consider it when high precision should be achieved.

Figs. 2 and 3 illustrate the gray level marker or "G-marker" proposed earlier by the authors. By using such type of markers - depending on the number of gray levels involved in the marker - several thresholded images have to be processed, i.e. the centre of gravity for each marker in each thresholded image are determined and the corresponding results are averaged. Usually this is a time consuming process, thus the "G-marker" in this form is less utilizable in real-time applications.

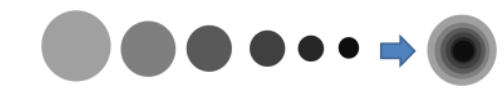

Figure 2

Illustration of the gray-level marker ("G-maker")

To minimize the errors more efficiently and speedup the processing - based on numerous analyses - a new type of marker, the so called "new G" marker has been proposed by the authors (see Figs. 4). The marker is composed from concentric circles of different gray-levels similarly to its previous version. However, in this case the ratios of radiuses are different. The number of composing circles is less than previously and their radiuses are closer to the maximal one. The multi-stage binarization of this improved "G-marker" may contribute to realization of realtime applications. Based on our analysis, the proposed marker showed more robustness to noise overall. 


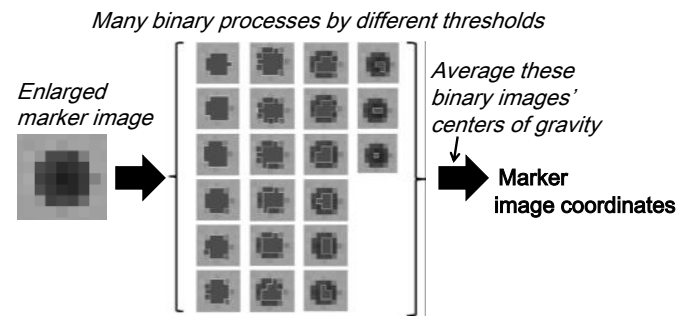

Figure 3

Illustration of marker extraction by thresholding. By modifying the threshold, the shape and size of markers will change accordingly. In order to reduce the error, the centers of gravities of corresponding markers are averaged
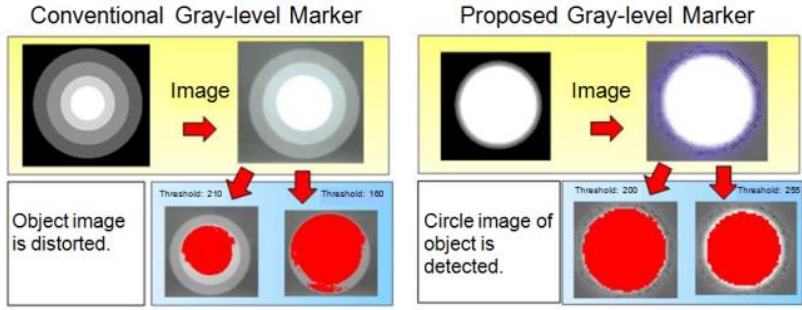

Figure 4

Illustration of the proposed gray-level marker and its efficiency

After the central point of each marker has been estimated, the correspondence problem has to be solved and finally based on triangulation the $3 \mathrm{D}$ coordinates are reconstructed. For the calibration of the cameras, the direct linear transformation (DLT) method was used [5].

\subsection{Compensation of Camera Distortions}

Almost each CCD (CMOS) image sensor is more or less sensitive to temperature variations, which has negative effect on the accuracy of the measurement. This phenomena can easily be recognized by placing a stable pattern composed for example from equidistant circular markers and starting to record the pattern by a camera. As the temperature in the device increases, difference in the sequentially acquired images can be observed. The detected centers fluctuate according to the temperature variation and depending on the location of the marker in the image. Various cameras of different types as well as their specifications were carefully inspected and the observed fluctuation as well as the influence of the pixel location on this variation have been identified for each type. According to the obtained data, the observed error is crucial when high accuracy should be achieved. As the temperature of the device becomes almost stable (according to our experiments this is few minutes after starting the recording) the fluctuation will remain in the range of \pm 0.17 pixel. 


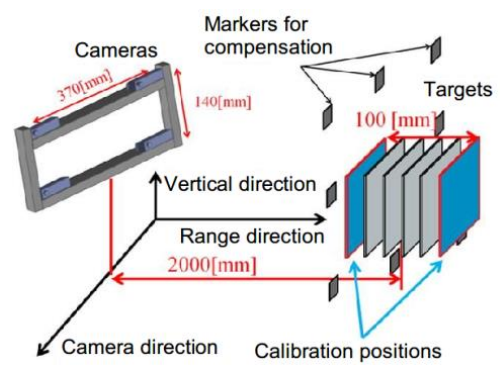

(a) Setup of the experimental system

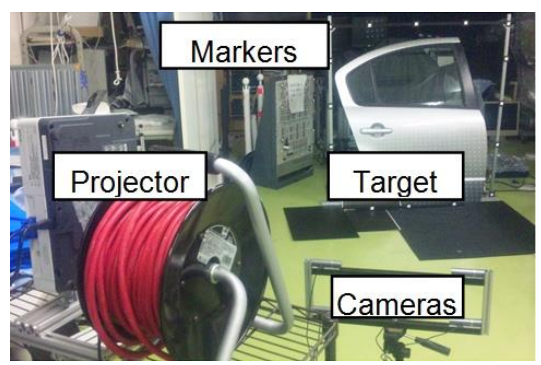

(b) Photo of the experimental system

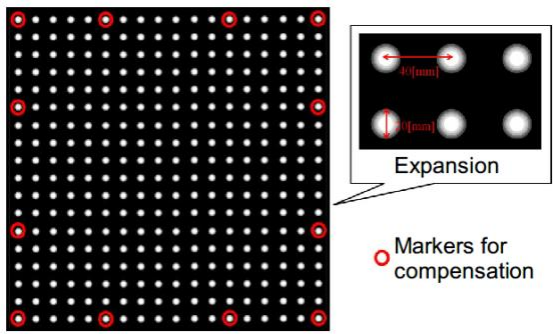

(c) Projector pattern used for error compensation

Figure 5

Compensation of the error caused by the temperature variation of the CCD (CMOS) sensor. The setup of the system (a), the photo of the system (b) and the pattern used for the compensation (c).

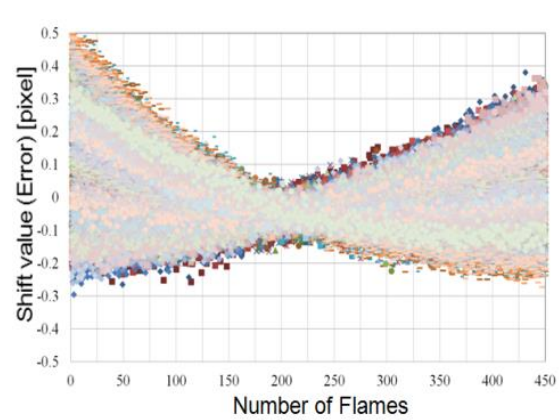

(a)

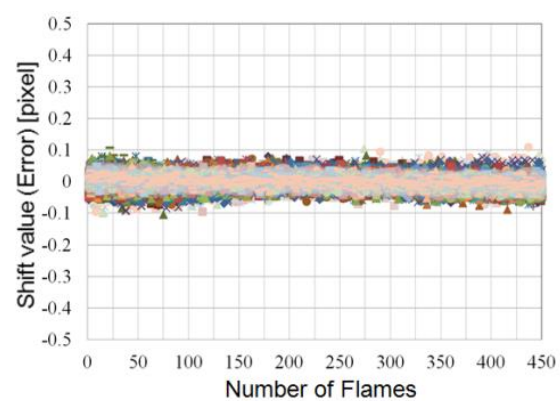

(b)

Figure 6

The measured variation in pixels: before compensation (a), after compensation by using the proposed method (b)

The minimal variation can be observed near the image centre and it increases according to the distance from it. The measured maximal variation was approximately the half of the pixel size that cannot be disregarded in high precision 3D measurement. 
Based on the obtained data, the authors proposed a method to compensate the observed variation with the help of a calibration pattern illustrated by Fig. 5c.

As compensation technique for sensor distortion by heat the following equation was proposed and validated:

$$
\left[\begin{array}{c}
X \\
Y
\end{array}\right]=\left[\begin{array}{lllllllllll}
C_{11} & C_{12} & C_{13} & C_{14} & C_{15} & C_{16} & C_{17} & C_{18} & C_{19} & C_{110} \\
C_{21} & C_{22} & C_{23} & C_{24} & C_{25} & C_{26} & C_{27} & C_{28} & C_{29} & C_{210}
\end{array}\right]\left[\begin{array}{c}
x \\
y \\
x^{2} \\
x y \\
y^{2} \\
x^{3} \\
x^{2} y \\
x y^{2} \\
y^{3} \\
1
\end{array}\right],
$$

where $x, y$ stand for the marker coordinates before compensation while $X$ and $Y$ represent the coordinates of the marker after compensation. The $C_{i j}$ values represent the compensation parameters.

In order to reduce the influence of the temperature change the camera temperature should be stabilized after startup.

During the calibration process based on numerous measurements first the characteristics of the fluctuation corresponding to the designated markers (red circles) in the pattern were identified (see Fig. 6a). By using such pattern the variation could efficiently be compensated. The achieved results can be followed in Fig. 6.

\subsection{Fast Search for Corresponding Points}

For cases when almost plain targets have to be measured, e.g. car door, engine hood, etc., a novel approach has been proposed by the authors to ensure the fast matching of corresponding markers. Fig. 7 illustrates the main concept of the matching principle. It can easily be recognized that an incorrectly identified marker yields a line of sight not crossing or being relatively distant from the intersection point of the other lines, i.e. the lines of sight corresponding to the correctly identified markers in the image planes of the other cameras.

There are many systems where the identification problem is solved by using active markers [13], structured lighting (3D scanning) [9] [14], etc. By these systems it is not necessary to search along epipolar lines and examine the neighboring pixels to find the corresponding one, so they are fast and robust. The proposed system has these properties, as well. The accuracy achieved by applying the above-described procedure has been compared to the case when epipolar line based searching technique was applied. 


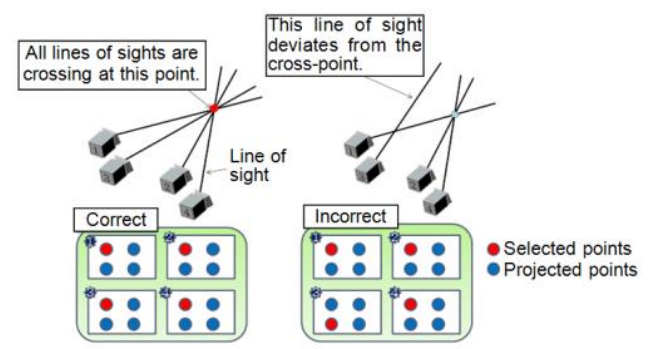

Figure 7

Successful identification of corresponding circles (left); incorrect identification of corresponding circles (right)

Fig. 8 shows the measured data obtained by the two different approaches. The accuracy achieved by the proposed technique is significantly higher than in case of applying the epipolar geometry. Due to the low complexity of the approach, the system can operate in real-time.
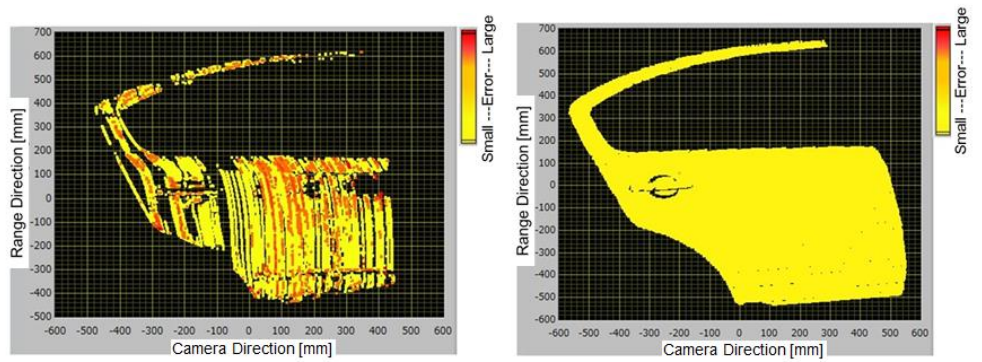

Figure 8

Searching for corresponding markers: the epipolar line based approach (left), the proposed approach (right)

The limitation of this matching technique is related to the shape characteristics of the target, i.e. the correspondence problem can be solved for limited depth changes only. The primary goal as already mentioned was to ensure the real-time measurement of car parts like door or engine hood, in which cases the requirements related to depth changes of the target are fulfilled.

After the identification of markers the 3D coordinates based on triangulation are estimated. Afterwards the projected pattern or the target is shifted and the whole procedure, i.e. detection, identification, reconstruction is repeated. Finally, a dense point cloud is obtained. In order to be able to measure larger depth differences, the density of the projected markers should be decreased. However, this would cause an increase in the duration of the whole reconstruction. This is because more shifts would be necessary to obtain a point cloud of same density. In the practical use, e.g. imagine the press line in the factory the targets are moved slowly to the next stages of processing. In this case the proposed system has the possibility to offer a dense point cloud, because the movement of the target is equivalent to the shift of the projected pattern. 


\section{Measurement Results}

\subsection{Target Measurement}

The efficiency of the proposed techniques and the effectiveness of the marker have been validated through various measurements and consequent analysis. During the tests, targets selected by the factory were measured. First of all the 3D reconstruction of a car door has been performed in real-time. Due to its size, it was challenging to perform its real-time reconstruction at once with an accuracy of few dozen microns (see below the details). Based on the achieved results, the application of the method seems to be promising in many industry related fields.

\subsection{Verification of the Measurement Accuracy}

In Fig. 9 the proposed gray level markers together with the achieved measurement accuracy can be followed. The target was a plain, non-painted aluminum plate (Fig. 9 (a)). The experimental setup can be followed in Fig. 5. During the experiment the location of each marker was examined. The error of the measurement was in the range $\pm 0.05 \mathrm{~mm}$.

Due to reflectance properties of metal usually it is difficult to measure non-painted metal plates with high precision. The achieved results show that the proposed markers are suitable for inspecting the car body parts during the production.
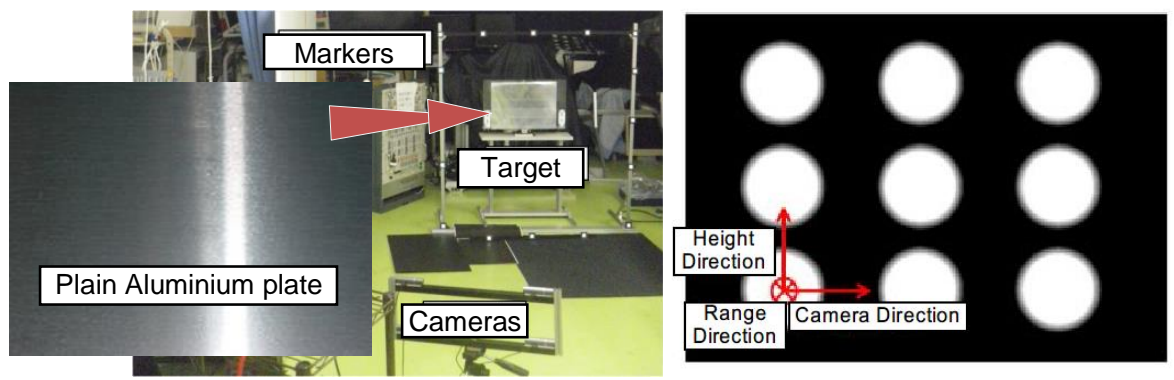

(a) Experimental setup: the targeted plate and the projected markers

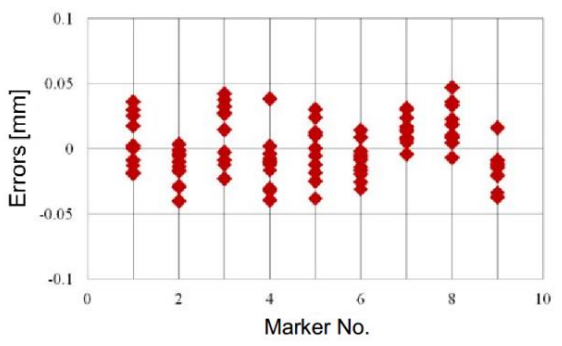

(b) Measurement error

Figure 9

Accuracy test of the marker central point estimation 


\subsection{An Example of Shape Measurement}

Fig. 10 shows the results obtained by using the proposed approach. The target was a painted car door. In order to obtain a dense point cloud the projected markers had to be shifted in horizontal and vertical directions, as well. Fig. 10 (a) and (b) show the image of the target and its reconstructed model, respectively. Fig. 10 (c) shows the measured target from an oblique view. In this latter figure, a small rectangular segment is expanded twice to visualize the regularity of measured points. The specifications of the proposed measurement system can be followed in Table 1.

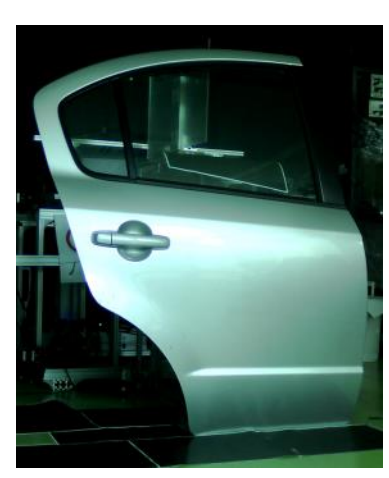

(a) Image of the target (car door)

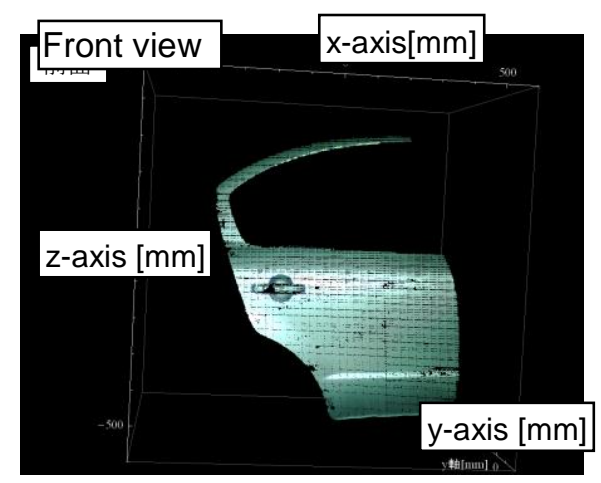

(b) Measured target (Front view)

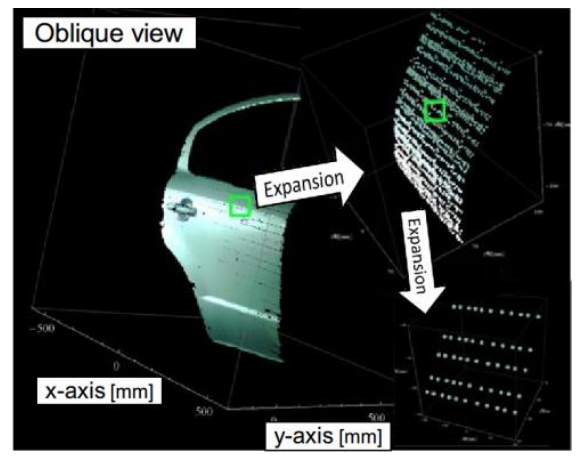

(c) Measured target (Oblique view with an expanded segment)

Figure 10

Measurement of a real car door: The data falling into the indicated rectangle are illustrated in more detail 


\subsection{Application of the Image Based Shape Measurement Method}

In this section we point out the advantages of the image based shape measurement and show an application example.

Even if high-density point clouds are used, it is not trivial to extract feature-points from the point cloud. However images include features such as color, texture, etc., which can advantageously be applied to detect feature points on the target.

Product inspection and the related devices play important role in industrial applications where usually the result of the $3 \mathrm{D}$ measurement is compared to CAE data (CAD drawings). For such a comparison, feature points of drawings such as screw holes should be identified.

During this experiment the screw holes of the car door were measured. The results are shown in Fig. 11, where the 3D coordinates of four holes on the backside of the car door were measured. The absolute error was below $0.2 \mathrm{~mm}$.

During the practical use in the factories, usually both sides of the pressed car door are measured. Since the camera positions are known, the holes on the backside are effective clues to match the front side of the car door with the CAE data.

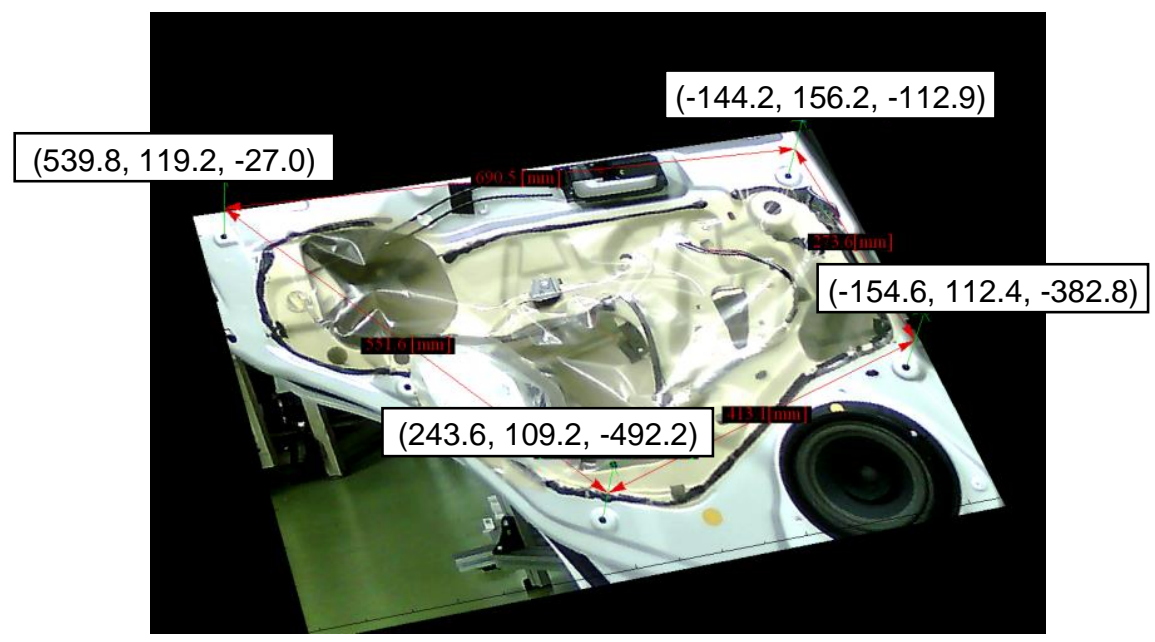

Figure 11

Testing the accuracy: measurement of a rectangular non painted aluminum plate attached to the car door 


\section{Summary}

\section{Discussions}

In Table 1 the features of the proposed method are compared to other shape measurement techniques. As each method has good and bad points, suitable methods to measure targets are still welcome.

The features of the proposed method can be followed in the left column. The main advantage of the approach is the flexibility and the high speed operation. As disadvantage the relatively low density of the resulted point cloud can be mentioned.

\section{Conclusions and Future Work}

In the paper, a real-time measurement system has been proposed for measurement of nearly plain car body parts. The main advantage of the system is the high accuracy (minimum about $0.05 \mathrm{~mm}$ ), the large measurement volume and the realtime processing. In order to achieve the mentioned features a new type of markers the so-called "new G" marker has been proposed by the authors.

Table 1

Comparison between the proposed method and other methods (representative value)

\begin{tabular}{|c|c|c|c|c|}
\hline & $\begin{array}{l}\text { Propose } \\
\text { method }\end{array}$ & $\begin{array}{c}\text { RANGE7 } \\
\text { (KONICA } \\
\text { MINOLTA } \\
\text { HOLDINGS, Inc.) }\end{array}$ & $\begin{array}{c}\text { ScanStation C10 } \\
\text { (Leica } \\
\text { Geosystems) }\end{array}$ & $\begin{array}{c}\text { TDS-0216D } \\
\text { (Pulstec Industrial } \\
\text { Co., Ltd.) }\end{array}$ \\
\hline $\begin{array}{c}\text { Measurement } \\
\text { area(horizonta } \\
\text { l) }\end{array}$ & $\begin{array}{l}\text { Approx. } 1500 \\
{[\mathrm{~mm}]}\end{array}$ & 79 or $267[\mathrm{~mm}]$ & $40[\mathrm{deg}]$ & $91[\mathrm{~mm}]$ \\
\hline $\begin{array}{l}\text { Measurement } \\
\text { area (vertical) }\end{array}$ & $\begin{array}{l}\text { Approx. } 1500 \\
{[\mathrm{~mm}]}\end{array}$ & 99 or $334[\mathrm{~mm}]$ & $40[\mathrm{deg}]$ & $80[\mathrm{~mm}]$ \\
\hline $\begin{array}{l}\text { Measurement } \\
\text { area (range } \\
\text { direction) }\end{array}$ & $1-5[\mathrm{~m}]$ & 450 or $800[\mathrm{~mm}]$ & $0.1-300[\mathrm{~m}]$ & $160-220[\mathrm{~mm}]$ \\
\hline Errors [mm] & 0.1 & 0.04 & 6 & 0.025 \\
\hline $\begin{array}{l}\text { Number of } \\
\text { measurement } \\
\text { points }\end{array}$ & $\begin{array}{c}36,300 \text { [points] } \\
\text { with projected } \\
\text { marker images } \\
\text { shifted } \\
\end{array}$ & $\begin{array}{c}\text { Over } 100 \\
\text { million[points] }\end{array}$ & $\begin{array}{c}\text { Max } \\
50,000[\text { points/s] }\end{array}$ & 200,000 [points] \\
\hline Scan time & $\begin{array}{c}1 / 30 \text { [sec.] } \\
(1 \text { projected } \\
\text { marker image })\end{array}$ & $\begin{array}{c}\text { Approx. } 2 \text { [sec.](1 } \\
\text { scan })\end{array}$ & $10[\mathrm{~min}]$. & $16[\mathrm{sec}]$. \\
\hline $\begin{array}{c}\text { Target } \\
\text { move/still }\end{array}$ & Move and Still & Still & Still & Still \\
\hline Advantages & $\begin{array}{l}\text { Wide area, } \\
\text { Real-time }\end{array}$ & $\begin{array}{c}\text { Standard system } \\
\text { of 3D shape } \\
\text { measurement }\end{array}$ & $\begin{array}{l}\text { For surveying } \\
\text { outdoors }\end{array}$ & $\begin{array}{l}\text { High precision, } \\
\text { Possible of Metal } \\
\text { surface shape } \\
\text { measurement }\end{array}$ \\
\hline
\end{tabular}


During the measurement, the errors caused by the temperature variation of the camera were taken into account, as well. Due to the temperature the internal properties of the camera, especially the properties of the CCD (CMOS) image sensor are varying. The authors of this paper proposed a method for reducing the effect of this kind of error source. Accordingly an accuracy of few dozen microns could be achieved. As future work, the system is planned to be extended by mirrors in order to ensure the measurement of hidden parts, as well. The method can efficiently be applied to support cliff failure prediction by measuring the cliff deformation. In this case, the markers are attached to the cliff. Furthermore, thermal cameras are going to be applied to add an additional dimension, i.e. temperature to the measured data, as well as to ensure the measurement of transparent surfaces. In order to model the influence of the temperature variation of the camera more accurately and reduce the related errors, we are planning to measure the temperature distribution and its variation more accurately by high sensitive thermal cameras and create the corresponding mathematical models.

\section{Acknowledgement}

A part of this research was supported by Grants-in-Aid for Scientific Research, MEXT (No. 22560416) and partly realized through the assistance of the European Union, with the co-financing of the European Social Fund TÁMOP-4.2.1.B11/2/KMR-2011-0001.

\section{References}

[1] M. Young, E. Beeson, J. Davis, S. Rusinkiewicz and R. Ramamoorthi, "Viewpoint-Coded Structured Light", IEEE Computer Society Conference on Computer Vision and Pattern Recognition, pages 1-8, June 2007

[2] E. Lilienblum, B. Michaelis, "Optical 3D Surface Reconstruction by a Multi-Period Phase Shift Method," Journal of Computers, Vol. 2, No. 2, pp. 73-83, 2007

[3] Yan Cui, Schuon, S., Chan, D., Thrun, S., Theobalt, C., "3D Shape Scanning with a Time-of-Flight Camera," 2010 IEEE Conference on Computer Vision and Pattern Recognition (CVPR), pp. 1173-1180, 13-18 June 2010

[4] N. Karpinsky, S. Zhang, "High-Resolution, Real-Time 3D Imaging with Fringe Analysis," Journal of Real-Time Image Processing, SpringerVerlag, Vol. 7, Issue 1, pp. 55-66, 2010

[5] Shapiro, R., "Direct Linear Transformation Method for Three-Dimensional Cinematography," Research Quarterly, 49(2), pp. 197-205, May 1978

[6] H. Faraji, W. J. MacLean, "CCD Noise Removal in Digital Images," IEEE Transactions on Image Processing, Vol. 15, No. 9, pp. 2676-2685, Sept. 2006 
[7] Y. Wonpil; Y. Chung; "An Embedded Camera Lens Distortion Correction Method for Mobile Computing Applications," IEEE International Conference on Consumer Electronics, pp. 400-401, 17-19 June 2003

[8] R. Cucchiara, C. Grana, A. Prati, R. Vezzani, "A Hough Transform-based Method for Radial Lens Distortion Correction,", In Proc. of the $12^{\text {th }}$ International Conference on Image Analysis and Processing, pp. 182-187, 17-19 Sept. 2003

[9] Kai Liu, Yongchang Wang, Daniel L. Lau, Qi Hao, and Laurence G. Hassebrook, "Dual-Frequency Pattern Scheme for High-Speed 3-D Shape Measurement," Optics Express, 18(5), pp. 5229-5244, 2010

[10] Gokturk, S. B.; Yalcin, H.; Bamji, C., "A Time-Of-Flight Depth Sensor System Description, Issues and Solutions," IEEE Conference on Computer Vision and Pattern Recognition Workshop, p. 35, June 2004

[11] Grubisic, I.; Gjenero, L.; Lipic, T.; Sovic, I.; Skala, T., "Active 3D Scanning-based 3D Thermography System and Medical Applications," In Proc. of the $34^{\text {th }}$ IEEE International Convention, pp. 269-273, 23-27 May 2011

[12] Li Yue, Xianyong Liu, "Application of 3D Optical Measurement System on Quality Inspection of Turbine Blade," 2009. IE\&EM '09. 16th International Conference on Industrial Engineering and Engineering Management, pp. 1089-1092, 21-23 Oct. 2009

[13] Janssen, R.; Lou, E.; Durdle, N.G.; Raso, J.; Hill, D.; Liggins, A.B.; Mahood, S., "Active Markers in Operative Motion Analysis," IEEE Transactions on Instrumentation and Measurement, Vol. 55, No. 3, pp.854859, June 2006

[14] Gupta, M.; Agrawal, A.; Veeraraghavan, A.; Narasimhan, S.G., "Structured Light 3D Scanning in the Presence of Global Illumination," 2011 IEEE Conference on Computer Vision and Pattern Recognition (CVPR), pp. 713720, 20-25 June 2011 